\title{
Cancer Control
}

National Cancer Institute

\section{Source}

National Cancer Institute. Cancer Control. NCI Thesaurus. Code C15971.

Integ rated program having an objective of reducing risk, incidence, and mortality from cancer and to enhancing the quality of life for cancer survivors. Realized through an orderly sequence of measures from genetic, epidemiologic, behavioral, social, applied, and surveillance cancer research to the development and implementation of effective interventions, and monitor and analysis of cancer trends in all segments of the population. 